\title{
miR-205 suppresses the proliferative and migratory capacity of human osteosarcoma MG-63 cells by targeting VEGFA
}

This article was published in the following Dove Press journal:

OncoTargets and Therapy

16 September 2015

Number of times this article has been viewed

\section{Li Wang \\ Minhong Shan \\ Fengyi Yang \\ Yang Liu \\ Hongxia Qi \\ Lijuan Zhou \\ Lirong Qiu \\ Yanshuang $\mathrm{Li}$}

Department of Operating Room I, Second Affiliated Hospital of Harbin Medical University, Harbin, Heilongjiang Province, People's Republic of China
Correspondence: Yanshuang Li 246 Xuefu Road, Nangang District, Harbin I50086, Heilongjiang Province, People's Republic of China

Tel +86 45I 86605222

Fax +8645 I 86605222

Email liyshuangI23_doc@|26.com
Background: Osteosarcoma (OS) is the most common primary bone malignancy in children and young adults. MiR-205 has been reported to be negatively correlated with the proliferation and metastasis of many types of cancer, while its effects on the malignant phenotype of OS are unclear.

Methods: Using TaqMan RT polymerase chain reaction analysis, we firstly explored the expression of miR-205 in a panel of OS cell lines. As the expression of miR-205 was significantly decreased in these cell lines, we sought to compensate for its loss by transfection of exogenous miR-205 mimic into MG-63 cells. To further understand the role of miR-205 in OS, we investigated the effects of miR-205 on the proliferation, migration, and invasion of MG-63 cells, and further explored the mechanisms that might be involved.

Results: We found that miR-205 was consistently suppressed in OS cells when compared with the normal human osteoblast (NHOst) cell line. Restored expression of miR-205 in the OS (MG-63) cell line significantly inhibited cell proliferation, migration, and invasion. Moreover, bioinformatic prediction suggested that vascular endothelial growth factor $\mathrm{A}(V E G F A)$ was the target oncogene for miR-205 in OS cells. Further quantitative RT polymerase chain reaction and Western blot assays identified that overexpression of miR-205 suppressed expression of VEGFA mRNA and protein. Restored expression of VEGFA in MG-63 cells previously treated with miR-205 mimic could partially abolish miR-205-mediated suppression of proliferation and invasion of these cells.

Conclusion: Collectively, these data suggest that miR-205 might function as a tumor suppressor in OS by, at least partially, targeting VEGFA.

Keywords: miR-205, osteosarcoma, vascular endothelial growth factor A, metastasis

\section{Introduction}

Osteosarcoma (OS) is the most common primary bone malignancy in children and young adults, and accounts for approximately $60 \%$ of malignant bone tumors in the first 2 decades of life. ${ }^{1}$ Pulmonary metastasis is the most common cause for cancer-related death. ${ }^{2}$ For patients with no metastatic disease at diagnosis, the 5-year survival rate is $60 \%-70 \%$. ${ }^{3}$ However, for patients who present with metastatic disease, the outcome is far worse, with less than 30\% survival. Hence, identification of new molecules involved in tumor metastasis is of crucial importance to reduce the morbidity and mortality of this devastating disease.

Nowadays, a group of endogenous, small, non-coding RNAs (known as miRNAs) are emerging as novel biomarkers of disease. ${ }^{4}$ By partially complementing the 3 -untranslated region of specific mRNAs, regulating its translational efficiency, or cleavage of targets, miRNAs induce gene silencing of various target mRNAs, ${ }^{5}$ and then 
participate in a variety of biological processes, including cell proliferation, apoptosis, differentiation, and metabolism. ${ }^{6}$

The effects of dysregulated miRNAs on the initiation and progression of cancer have been widely reported. ${ }^{7}$ Some of these miRNAs have been associated with tumor invasion and metastasis. ${ }^{8}$ MiR-205 is an miRNA that is highly conserved in a number of species, and has been reported to have a close relationship with metastasis in many cancers. ${ }^{9}$ For example, miR-205 was reported to be significantly downregulated in gastric cancer tissue when compared with normal gastric tissue, and was associated with negative clinical and pathological characteristics in patients. ${ }^{10}$ Furthermore, by counteracting epithelial-mesenchymal transition, expression of miR-205 is inversely associated with aggressive behavior of malignant mesothelioma and suppresses its proliferation and invasion. ${ }^{11}$ However, the effects of miR-205 on the metastasis of OS cells and the molecular mechanisms involved are largely unknown.

In this study, we demonstrated that expression of miR-205 was suppressed in a panel of OS cells, and ectopic miR-205 expression suppressed migration and invasion of OS cells in vitro. Furthermore, vascular endothelial growth factor A (VEGFA) was identified to be a target gene of miR-205 in OS cells, and restoration of $V E G F A$ expression partially abolished miR-205-mediated suppression of cell migration and invasion in OS cells, suggesting that miR-205 might function as a tumor suppressor in OS by, at least partially, targeting VEGFA.

\section{Materials and methods Cell culture and cell transfection}

A panel of human OS (HOS, SaOS-2, U2OS, and MG-63) cell lines and a normal osteoblast (NHOst) cell line were obtained from the American Type Culture Collection (Manassas, VA, USA). The cells were maintained in Dulbecco's Modified Eagle's Medium (Gibco, Life Technologies, Darmstadt, Germany) or Roswell Park Memorial Institute 1640 medium supplemented with $10 \%$ fetal bovine serum (PAA, Pasching, Austria), streptomycin $100 \mu \mathrm{g} / \mathrm{mL}$, and penicillin $100 \mathrm{U} / \mathrm{mL}$. Cultures were incubated in a humidified atmosphere of $5 \%$ $\mathrm{CO}_{2}$ at $37^{\circ} \mathrm{C}$. MiR-205 and scramble mimic were purchased from Dharmacon (Austin, TX, USA), and then transfected into OS cells to a final concentration of $50 \mathrm{nM}$ by Dharmafect 1 (Dharmacon) according to the manufacturer's instructions.

\section{RNA extraction and reverse transcription and quantitative real-time PCR}

Total RNA was extracted from cells using the TRIzol reagent (Invitrogen, Carlsbad, CA, USA) according to the manufacturer's instructions. First, total RNA was reversely transcribed using a
First-Strand cDNA Synthesis kit (Invitrogen). Quantitative realtime polymerase chain reaction (RT-PCR) was then performed using Quanti-Tect SYBR Green PCR mixture on an ABI Prism 7900 Sequence Detection System (Applied Biosystems, Carlsbad, CA, USA). Specific primers for reverse transcription and RT-PCR are shown in Table 1. Expression levels of U6 and glyceraldehyde-3-phosphate dehydrogenase were used as the internal control for miRNA and mRNA, respectively. PCR efficiencies were calculated with a relative standard curve derived from a complementary DNA mixture and gave regression coefficients $>0.95$. The relative expression levels were evaluated using the $2^{-\Delta \Delta C t}$ method. All experiments were repeated three times to reduce curve-derived variance.

\section{Cell proliferation assay}

Cell Counting Kit-8 (CCK-8; Dojindo, Kumamoto, Japan) assays was used for analysis of cell proliferation. MG-63 cells were seeded into 24 -well plates at $5 \times 10^{3}$ cells/well. The cells were then incubated in 10\% CCK- 8 and diluted in normal culture medium at $37^{\circ} \mathrm{C}$ until visual color conversion occurred. The absorbance in each well was measured with a microplate reader set at $450 \mathrm{nM}$ and $630 \mathrm{nM}$ at $0,24,48$, and 72 hours after transfection.

\section{Cell migration and invasion assay}

A Transwell device containing $8 \mu \mathrm{m}$ microporous membranes (Corning Incorporated, Corning, NY, USA) was placed in 24-well plates. The sides of the upper chamber of the basal membrane were coated with $5 \mathrm{mg} / \mathrm{mL}$ of Matrigel (BD Biosciences, Franklin Lakes, NJ, USA). Normal MG-63 cells or MG-63 cells transfected with miR-205 or scramble mimic were seeded into the upper chamber containing Dulbecco's Modified Eagle's Medium with $0.1 \%$ bovine serum albumin. Dulbecco's Modified Eagle's Medium containing 10\% fetal bovine serum served as the chemoattractant. The migration/ invasion assay was followed for 24 hours. Cells adhering to the lower surface were fixed and stained with $0.1 \%$ crystal violet and transferred to a microscope slide. The total number of invading cells was counted in six representative fields under microscopy (200× magnification).

\section{Vector construction and luciferase assays}

The whole $3^{\prime}$-UTR of the VEGFA gene was amplified from genomic DNA and cloned into the pGL-3 vector (Promega, Madison, WI, USA) immediately downstream of the Renilla luciferase gene. Mutations in the 3'-UTR of the VEGFA gene with the miR-205 target site deleted (MUT) were generated using the QuickChange Site-Directed Mutagenesis 
Table I Oligonucleotide primer sequences for PCR or reverse transcription

\begin{tabular}{ll}
\hline Gene & Primer sequence \\
\hline Primers for real-time PCR & \\
miR-205-sense & $5^{\prime}$-GCTCCTTCATTCCACCGG-3' \\
miR-205-antisense & $5^{\prime}$-CAGTGCAGGGTCCGAGGT-3' \\
U6-sense & $5^{\prime}$-CTCGCTTCGGCAGCACATATACT-3' \\
U6-antisense & $5^{\prime}$-ACGCTTCACGAATTTGCGTGTC-3' \\
VEGFA-sense & $5^{\prime}$-AAGGAGGAGGGCAGAATCAT-3' \\
VEGFA-antisense & $5^{\prime}$-GCACACAGGATGGCTTGAA-3' \\
GAPDH-sense & $5^{\prime}$-TCAACGACCACTTTGTCAAGCTCA-3' \\
GAPDH-antisense & $5^{\prime}$-GCTGGTGGTCCAGGGGTCTTACT-3' \\
Primers for reverse transcription & \\
miR-205 & \\
U6 & $5^{\prime}$-GTCGTATCCAGTGCAGGGTCCGAGGTATTCGCACTGGCAGACT-3' \\
VEGFA and GAPDH & $5^{\prime}$-AAAATATGGAACGCTTCACGAATTTG-3' \\
Primers for VEGFA PCR amplifying & $5^{\prime}$-TTTTTTTTTTTTTTTTTT-3' oligo(dT) \\
VEGFA-F & \\
VEGFA-R & $5^{\prime}$-CCCAAGCTTCTGACGGACAGACAGACA-3' \\
Primers for VEGFA luciferase reporter & $5^{\prime}$-CCGCTCGATCAGTCTTTCCTGGTGAGA-3' \\
VEGFA-F & \\
VEGFA-R & $5^{\prime}$-AGGAAAGACTGATACAGAACGA-3' \\
\hline
\end{tabular}

Abbreviations: GAPDH, glyceraldehyde-3-phosphate dehydrogenase; PCR, polymerase chain reaction; VEGFA, vascular endothelial growth factor A; F, forward; R, reverse.

kit (Stratagene, La Jolla, CA, USA). A luciferase reporter construct containing the miR-205 consensus target sequence served as the positive control, and the pRL-TK vector were used as positive and internal controls, respectively. Approximately $1 \times 10^{5}$ MG-63 cells per well were seeded into 24-well plates for 24 hours before transfection. Cells were cotransfected with $50 \mathrm{ng}$ of pGL-3 firefly luciferase reporter, $10 \mathrm{ng}$ pRL-TK Renilla luciferase reporter, and $50 \mathrm{nM} \mathrm{miR-205}$ or scramble mimic using Lipofectamine ${ }^{\mathrm{TM}} 2000$ (Invitrogen). Cell lysates were prepared using passive lysis buffer (Promega) 48 hours after transfection, and luciferase activity was measured using a dual-luciferase reporter assay (Promega). Results were normalized to Renilla luciferase.

\section{Rescue assays for VEGFA gene expression}

The full length $V E G F A$ gene open reading frame were amplified by PCR and then cloned into a pCDNA-3.1 construct to generate the pCDNA-3.1-VEGFA construct. The empty pCDNA-3. 1 construct was used as the control. MG-63 cells were first transfected with miR-205 or scramble mimic $(60 \mathrm{nM})$ in 6-well plates. After 24 hours of culture, the MG-63 cells were cotransfected with miR-205 mimic $(30 \mathrm{nM})$ and $2.0 \mu \mathrm{g}$ of either pcDNA-3.1-VEGFA or pcDNA-3.1 constructs. The cells were harvested at predetermined intervals and assays as necessary.

\section{Western blot analysis}

For the Western blot assay, cells were harvested in ice-cold phosphate-buffered saline 48 hours after transfection and lysed on ice in cold-modified radioimmunoprecipitation buffer supplemented with protease inhibitors. The protein concentration was determined using a bicinchoninic acid protein assay kit. Equal amounts of protein were analyzed by sodium dodecyl sulfate polyacrylamide gel electrophoresis. Gels were electroblotted onto nitrocellulose membranes (Millipore, Billerica, MA, USA). Membranes were blocked for 2 hours with $5 \%$ non-fat dry milk in Tris-buffered saline containing $0.1 \%$ Tween- 20 , and incubated at $4^{\circ} \mathrm{C}$ overnight with primary antibody, VEGFA and GAPDH (Cell Signaling Technology, Danvers, MA, USA). Detection was performed after peroxidase-conjugated secondary antibodies using an enhanced chemiluminescence system (Millipore).

\section{Statistical analysis}

All experiments were repeated independently at least three times. Data are expressed as the mean \pm standard deviation of repeated experiments. The statistical analysis was carried out using Statistical Package for the Social Sciences version 15.0 software (SPSS Inc, Chicago, IL, USA). The Student's $t$-test (two-tailed) was performed to analyze the data. $P$-values $<0.05$ were considered to be statistically significant.

\section{Results}

\section{MiR-205 was suppressed in a panel of OS cells}

Although miR-205 has been reported to be a tumor suppressor in various cancers, its exact role in OS has not been 
verified as yet. In an attempt to explore its expression and significance in osteocarcinogenesis and disease progression, we firstly detected the expression of miR-205 in a panel of OS cell lines using TaqMan quantitative RT-PCR. As shown in Figure 1, compared with normal human osteoblast (NHOst) cells, expression of miR-205 was consistently downregulated in four OS cell lines (HOS, SaOS-2, U2OS, and MG-63, Figure 1). These data suggest that alteration of miR-205 might function as a tumor suppressor in OS cells.

\section{MiR-205 suppresses proliferation, migration, and invasion of MG-63 cells}

To explore further the effects of miR-205 on OS cells with a malignant phenotypes, MG-63 cells were treated with miR-205 or scramble mimic. Upon transfection, the intracellular level of miR-205 was about 80-fold higher in MG-63 cells transfected with miR-205 mimic relative to the scramble control group (Figure 2A). Next, we explored the effects of miR-205 on cell proliferation using the CCK-8 assay. As shown in Figure 2B, treatment with miR-205 significantly suppressed proliferation of MG-63 cells.

Cell migration and invasion promote tumor metastasis, which is the major cause of death in patients with OS. Thus, we explored the effects of miR-205 on migration and invasion of MG-63 cells using Transwell assays with or without Matrigel, respectively. Transwell assays without Matrigel showed that restoration of miR-205 expression in MG-63 cells resulted in a significant reduction in the number of cells passing through the chambers compared with the scramble group ( $168 \pm 30$ cells vs $62 \pm 15$ cells; $P<0.05$, Figure 2 C). Before the invasion assays, the chambers were coated with Matrigel, which mimics the extracellular matrix. A similar result was

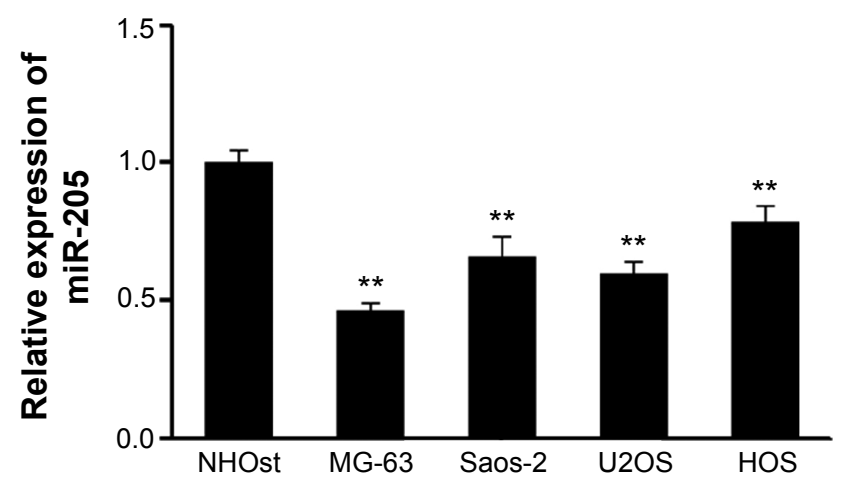

Figure I Expression of miR-205 in OS cell lines.

Notes: Expression of miR-205 in four OS cell lines (HOS, Saos-2, U2OS, and MG63) was analyzed using TaqMan quantitative RT polymerase chain reaction. The expression of miR-205 in these OS cell lines was under-regulated relative to the normal osteoblast $(\mathrm{NHOst})$ cell line. ${ }^{* * P}<0.01$ compared with normal osteoblast cells. The experiments were repeated five times.

Abbreviations: OS, osteosarcoma; RT. real-time. observed in the invasion assays. As shown in Figure 2D, overexpression of miR-205 could significantly suppress the number of cells passing through the chambers $(123 \pm 29$ cells vs $48 \pm 17$ cells; $P<0.05)$. These data indicate that miR-205 can efficiently repress cell motility and invasiveness of OS cells in vitro.

\section{VEGFA is a putative target gene of miR- 205 in MG-63 cells}

To explore the mechanisms involved in the suppressive effects triggered by miR-205 in OS cells, putative targets of miR-205 were searched for using prediction programs. Among the common predicted targets of miR-205, VEGFA was selected as an ideal candidate because of its overexpression in $\mathrm{OS}^{12}$ and its putative role as an oncogene in a number of cancers. As shown in Figure 3A, miR-205 has a predicted binding site in the 3'-UTR of VEGFA. Dual-luciferase assays were performed to determine if this site has a function in OS cells. The 3'-UTR of the VEGFA gene was cloned into a luciferase reporter vector, pGL-3, and the mutant construct with deletion of the putative binding site was used as a negative control. Both the wild-type pGL-3.1-VEGFA 3'-UTR construct and the mutant one were transiently transfected into MG-63 cells, and the transcription activity was measured. As expected, significant repression of luciferase activity was observed in MG-63 cells cotransfected with the pGL-3.1-VEGFA 3'-UTR construct and miR-205 mimic compared with the mutant construct group (Figure 3B), suggesting that miR-205 suppressed the transcriptional activity of the VEGFA gene. Consistent with the reporter assays, we observed that transfection with miR-205 decreased mRNA and protein expression of $V E G F A$ relative to the scramble groups (Figure $3 \mathrm{C}$ and $\mathrm{D}$ ). These findings suggest that $V E G F A$ is a direct target gene of miR-205 in OS cells.

\section{VEGFA is involved in miR-205-mediated suppression of proliferation and invasion of MG-63 cells}

$V E G F A$ is a key regulator of angiogenesis and plays an important role in regulation of tumor metastasis. However, whether it is involved in miR-205-mediated suppression of migration and proliferation of MG-63 cells remains unclear. Thus, a "rescue" methodology was adopted to examine the functional relevance of interaction between miR-205 and VEGFA in MG-63 cells. A new construct containing the full open reading frame of the VEGFA gene was generated. MG-63 cells were then cotransfected with miR-205 or scramble mimic and VEGFA or control constructs (pcDNA-3.1-VEGFA, or 
A
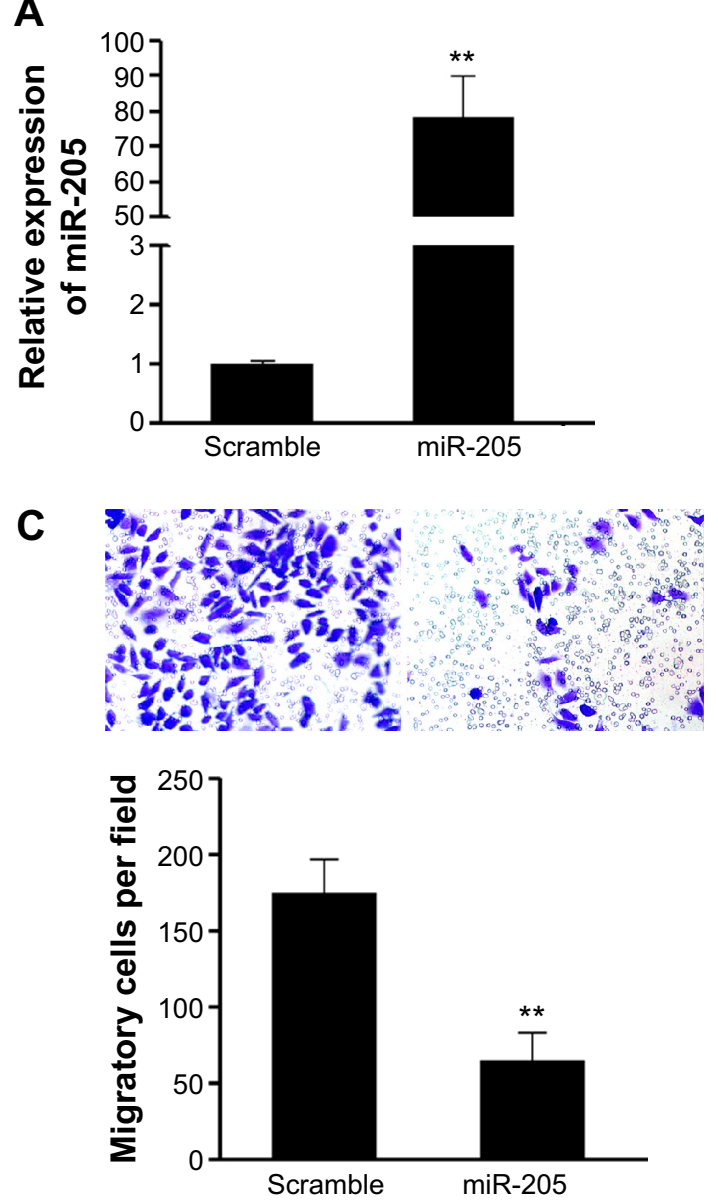

B

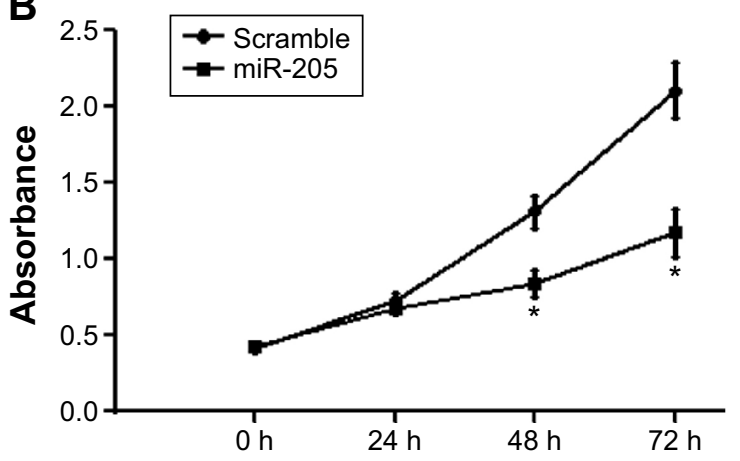

D
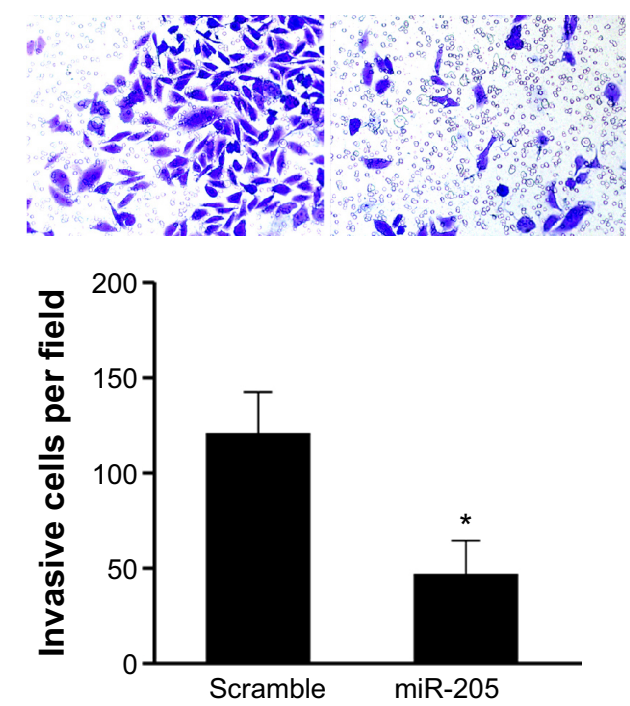

Figure 2 Effects of miR-205 on MG-63 cell proliferation and invasion.

Notes: (A) The level of miR-205 expression was detected in MG-63 cells upon transfection with miR-205 or scramble mimic by quantitative RT polymerase chain reaction. (B) A cell proliferation assay of MG-63 cells was performed after transfection with miR-205 or scramble mimic by using Cell Counting Kit-8. (C) Cell migration of MG-63 cells after transfection with miR-205 or scramble mimic was performed by wound healing assay and the relative ratio of wound closure per field is shown. (D) Cell invasion analysis of MG-63 cells upon transfection with miR-205 or scramble mimic was performed by Transwell assay, and the relative ratio of invasive cells per field is shown. $* P<0.05$, $* * P<0.01$ compared with scramble group. All experiments were repeated at least three times independently.

Abbreviation: $\mathrm{RT}$, real-time.

pcDNA-3.1), respectively. Upon transfection, expression of VEGFA was rescued when the VEGFA construct was transfected into MG-63 cells that had been treated with miR205 mimic for 24 hours (Figure 4A). In agreement with the restored expression of $V E G F A$, an increased cell proliferation rate was observed in MG-63 cells transfected with the VEGFA construct following treatment with the miR-205 mimic (Figure 4B). Moreover, upon transfection with the $V E G F A$ constructs, suppression of miR-205-mediated invasion (Figure 4C) in MG-63 cells was also partially abolished. These results demonstrate that $V E G F A$ is a functional target of miR-205, contributing to its role in miR-205-mediated repression of proliferation and invasion of OS cells.

\section{Discussion}

An increasing number of studies have characterized the expression profiles of miRNAs in tumors and have revealed that miRNAs are frequently dysregulated in tumor tissue., ${ }^{4,13}$ Depending on the genes they regulate, miRNAs function as oncogenes or tumor suppressors, and their mediation of the post-transcriptional gene regulation is emerging as an important epigenetic regulation mechanism. ${ }^{13-15}$ In regard to OS, a certain number of miRNAs have been reported to be involved in carcinogenesis, tumor progression, and prognosis. ${ }^{716,17}$ Therefore, understanding the physiological and disease-associated mechanisms of these small, singlestranded RNAs may provide us with new opportunities for diagnosis and therapy in the future.

MiR-205 is an miRNA that is highly conserved in a number of species. Although downregulation of miR-205 has been frequently observed in various types of cancer, ${ }^{18,19}$ its upregulation has also been noted in some types of cancer, ${ }^{20}$ suggesting that miR-205 may present different expression patterns in specific tissues, and may play different roles 
A
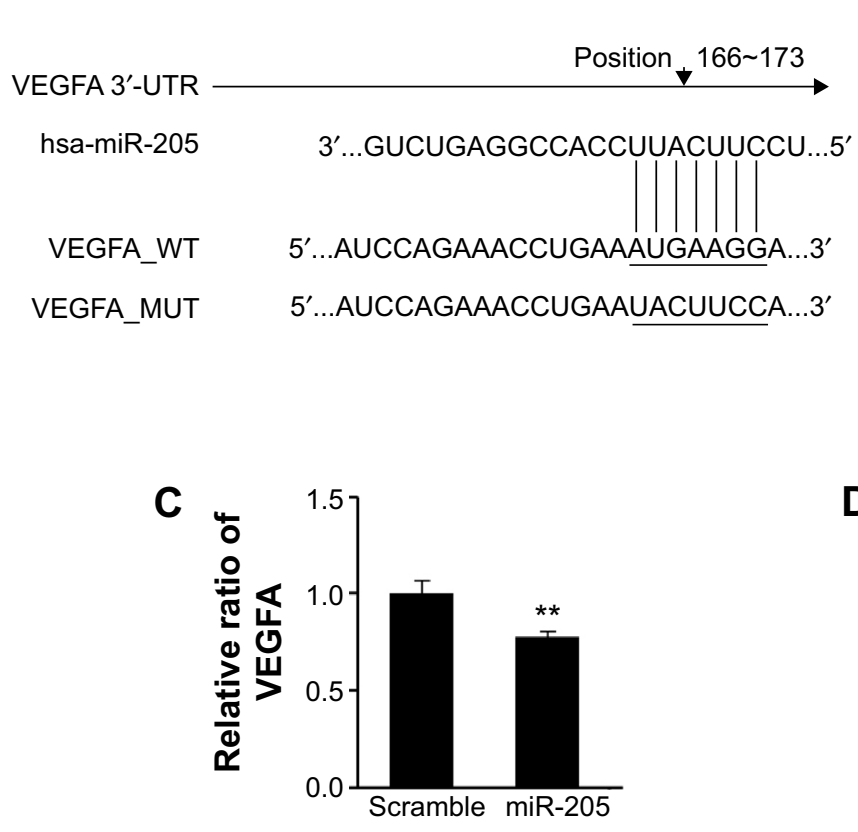

B

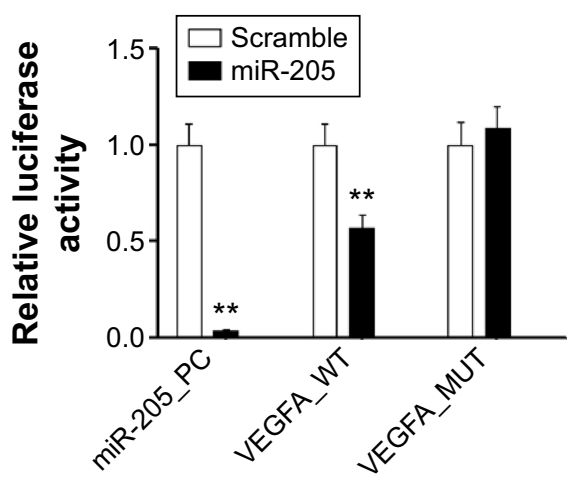

D

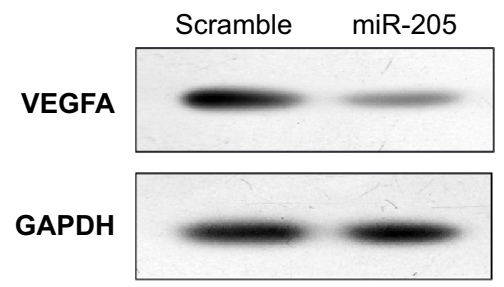

Figure 3 MiR-205 targets the VEGFA gene in osteosarcoma cells.

Notes: (A) Schematic representation of VEGFA 3'-UTR showing putative miR-205 target site. (B) Relative luciferase activity of the indicated VEGFA reporter construct in MG-63 cells, cotransfected with miR-205 or scramble mimic, is shown. (C) Quantitative RT polymerase chain reaction assay was performed to detect the expression of VEGFA upon transfection with miR-205 or scramble mimic. (D) Western blot analysis of the expression of VEGFA protein in MG-63 cells transfected with miR-205 or scramble mimic was performed. $* * P<0.01$, compared with scramble group. All experiments were repeated at least three times independently.

Abbreviations: VEGFA, vascular endothelial growth factor A; GADPH, glyceraldehyde-3-phosphate dehydrogenase; WT, wild-type; MUT, mutant; RT, real-time.

depending on the specific tumor microenvironment and target. ${ }^{21}$ For instance, miR-205 is highly expressed in a subpopulation of mouse mammary epithelial cell progenitors. By targeting the tumor suppressor PTEN, overexpression of miR-205 leads to an expansion of growth of progenitor cell and promotion of colony-forming potential, underlying its tumor initiation property. ${ }^{22}$ However, in melanoma specimens, ectopic expression of miR-205 leads to suppression of cell proliferation and induction of senescence by targeting E2F1-mediated Akt phosphorylation. ${ }^{23}$ One possible reason for this discrepancy might be that miR-205 forces an epithelial phenotype and is downregulated in tumors of mesenchymal organs. ${ }^{21}$ MiR-205 is located in the second intron of the LOC642587 locus in chromosome 1, and is highly expressed in the epidermis. ${ }^{24}$ It has been reported to orchestrate the process of epithelium morphogenesis during embryogenesis..$^{25}$ Moreover, its expression was observed to be specifically downregulated in cells that have undergone epithelial-mesenchymal transition, and is accompanied by a pronounced decrease in E-cadherin and an increase in $\mathrm{N}$-cadherin and fibronectin. ${ }^{26}$ In this study, we found that expression of miR-205 was consistently suppressed in a panel of OS cell lines, which are types of mesenchymal cells, when compared with the NHOst cell line, suggesting that miR-205 might function as a tumor suppressor in OS cells. Next, we examined its effects on migration and invasion of OS cells. As expected, restoration of miR-205 expression markedly inhibited cell proliferation, and suppressed migration and invasion of MG-63 cells, further indicating a tumor suppressor role of miR-205 in OS cells.

To further investigate the mechanisms of miR-205 in OS, we identified putative targets of miR-205 using prediction programs. Among these genes, VEGFA was identified, given that it has been shown to be closely related to carcinogenesis and metastasis of cancer. The target role of VEGFA was identified by luciferase reporter assays, as transfection of miR-205 caused a substantial reduction of luciferase activity by the luciferase expression constructs carrying the target $V E G F A$ fragment, and ectopic expression of miR-205 reduced the mRNA and protein levels of $V E G F A$ at the same time. Although $V E G F A$ has been demonstrated to be the target gene of miR-205 in breast cancer, ${ }^{27}$ this is the first time to validate miR-205 function as a tumor suppressor through targeting VEGFA in the progression of OS.

$V E G F A$ is a key regulator of angiogenesis, which is known to be a fundamental factor in local growth of tumors and progression to metastases. During the early hypoxic stage, cancer cells respond through activating multisignaling 

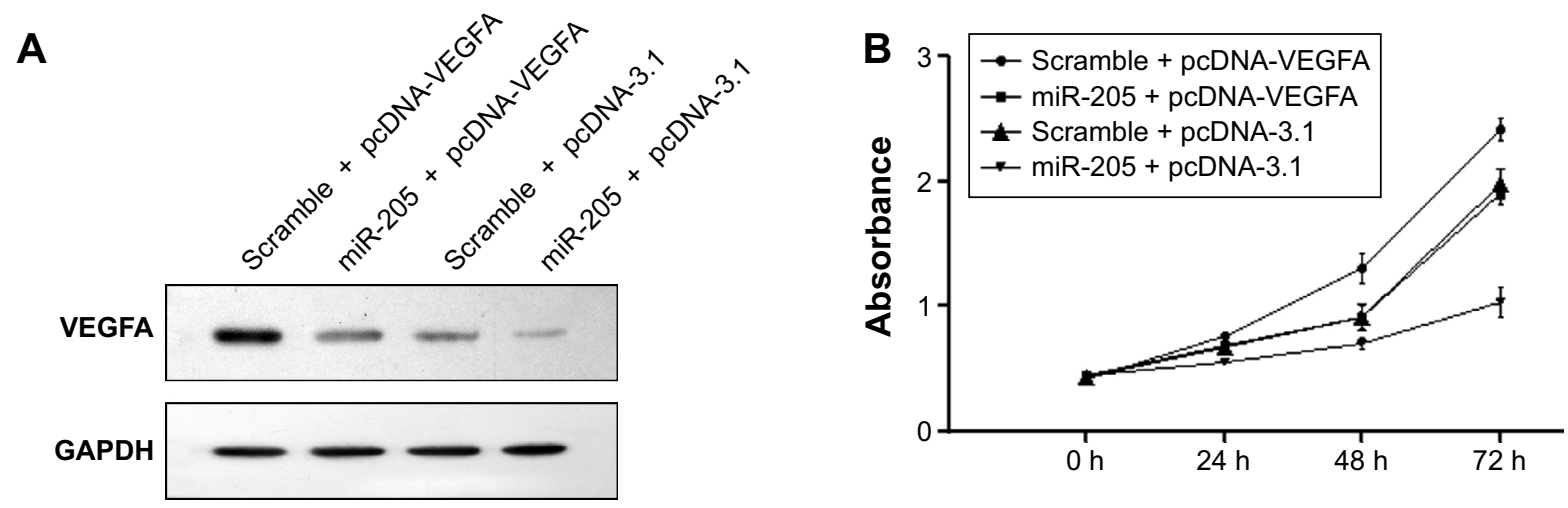

C

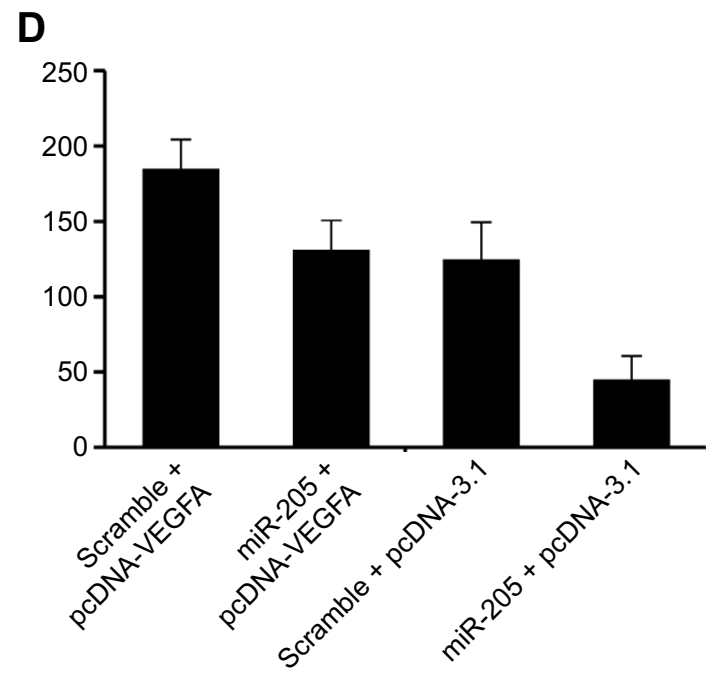

Figure 4 VEGFA is involved in miR-205-dependent control of MG-63 cell proliferation and invasion.

Notes: (A) The level of VEGFA protein expression was detected in MG-63 cells cotransfected with either miR-205 or scramble mimic and pcDNA-3.I-VEGFA or the pcDNA-3.I empty vector by Western blot assay. (B) Wound healing assays for MG-63 cells were performed to detect the effects of VEGFA on miR-205-mediated cell proliferation inhibition. (C, D) Cell invasion assays of MG-63 cells were performed to detect the effects of VEGFA on miR-205-mediated cell invasion inhibition. All experiments were repeated at least three times independently.

Abbreviations: VEGFA, vascular endothelial growth factor A; GADPH, glyceraldehyde-3-phosphate dehydrogenase.

pathways including VEGFA that induce cell proliferation, production of angiogenic factors, and formation of new endothelial cells in order to provide a vascular supply, ${ }^{28,29}$ which means VEGFA has an important role in tumor growth and metastasis. Suppression of $V E G F$ has been found to suppress migration and invasion of OS cells. Thus, antiVEGF treatment, such as with the anti-VEGF antibody bevacizumab, has been widely used to treat cancers in the clinical setting. ${ }^{30}$ High VEGF expression was reported to be associated with lower overall survival in OS. ${ }^{12}$ Yang et al also identified that elevated expression of VEGFA had a significant positive correlation with higher microvascular density and adverse correlation with tumor-free survival in OS. ${ }^{31}$ Although we did not explore the effects of VEGFA in OS cells, we found that restoration of VEGFA expression in OS cells previously treated with miR-205 could partially abolish miR-205-mediated suppression of cell proliferation and invasion, which indirectly identifies the oncogene role of VEGFA in OS and is involved in miR-205-mediated suppressive effects.

\section{Conclusion}

Taken together, our findings demonstrate that miR-205 acts as a tumor suppressor in OS MG-63 cells by targeting $V E G F A$. Reintroduction of miR-205 into MG-63 cells can downregulate $V E G F A$, which suppresses cell growth and inhibits cell migration and invasion, while overexpression of VEGFA could partially abolish the suppressive effects on cancer cells. This research not only helps us to understand the molecular mechanism of osteocarcinogenesis, but also provides a strong rationale to further investigate miR-205 as a potential biomarker and therapeutic target for OS.

\section{Acknowledgment}

This research was supported by the Second Affiliated Hospital of Harbin Medical University. 


\section{Author contributions}

All authors contributed toward data analysis, drafting and revising the paper and agree to be accountable for all aspects of the work.

\section{Disclosure}

The authors report no conflicts of interest in this work.

\section{References}

1. Ma O, Cai WW, Zender L, et al. MMP13, Birc2 (cIAP1), and Birc3 (cIAP2), amplified on chromosome 9, collaborate with p53 deficiency in mouse osteosarcoma progression. Cancer Res. 2009;69(6):2559-2567.

2. Ferguson WS, Goorin AM. Current treatment of osteosarcoma. Cancer Invest. 2001;19(3):292-315.

3. Bacci G, Ferrari S, Bertoni F, et al. Long-term outcome for patients with nonmetastatic osteosarcoma of the extremity treated at the Istituto Ortopedico Rizzoli according to the Istituto Ortopedico Rizzoli/ Osteosarcoma-2 Protocol: an updated report. J Clin Oncol. 2000;18(24): 4016-4027.

4. Kong YW, Ferland-McCollough D, Jackson TJ, Bushell M. microRNAs in cancer management. Lancet Oncol. 2012;13(6):e249-e258.

5. Bartel DP. MicroRNAs: genomics, biogenesis, mechanism, and function. Cell. 2004;116(2):281-297.

6. Chen K, Rajewsky N. The evolution of gene regulation by transcription factors and microRNAs. Nat Rev Genet. 2007;8(2):93-103.

7. Kafchinski LA, Jones KB. MicroRNAs in osteosarcomagenesis. $A d v$ Exp Med Biol. 2014;804:119-127.

8. Ell B, Kang Y. MicroRNAs as regulators of bone homeostasis and bone metastasis. Bonekey Rep. 2014;3:549.

9. Kalogirou C, Spahn M, Krebs M, et al. MiR-205 is progressively downregulated in lymph node metastasis but fails as a prognostic biomarker in high-risk prostate cancer. Int J Mol Sci. 2013;14(11):21414-21434.

10. Yin WZ, Li F, Zhang L, Ren XP, Zhang N, Wen JF. Down-regulation of microRNA-205 promotes gastric cancer cell proliferation. Eur Rev Med Pharmacol Sci. 2014;18(7):1027-1032.

11. Fassina A, Cappellesso R, Guzzardo V, et al. Epithelial-mesenchymal transition in malignant mesothelioma. Mod Pathol. 2012;25(1): $86-99$.

12. Chen D, Zhang YJ, Zhu KW, Wang WC. A systematic review of vascular endothelial growth factor expression as a biomarker of prognosis in patients with osteosarcoma. Tumour biology: the journal of the International Society for Oncodevelopmental Biology and Medicine. 2013;34(3):1895-1899.

13. Gurtan AM, Sharp PA. The role of miRNAs in regulating gene expression networks. Journal of Molecular Biology. 2013;425(19):3582-3600.

14. Cock-Rada A, Weitzman JB. The methylation landscape of tumour metastasis. Biology of the cell/under the auspices of the European Cell Biology Organization. 2013;105(2):73-90.

15. Bullock MD, Sayan AE, Packham GK, Mirnezami AH. MicroRNAs: critical regulators of epithelial to mesenchymal (EMT) and mesenchymal to epithelial transition (MET) in cancer progression. Biology of the cell/under the auspices of the European Cell Biology Organization. 2012;104(1):3-12.

OncoTargets and Therapy

\section{Publish your work in this journal}

OncoTargets and Therapy is an international, peer-reviewed, open access journal focusing on the pathological basis of all cancers, potential targets for therapy and treatment protocols employed to improve the management of cancer patients. The journal also focuses on the impact of management programs and new therapeutic agents and protocols on
16. Kobayashi E, Satow R, Ono M, et al. MicroRNA expression and functional profiles of osteosarcoma. Oncology. 2014;86(2):94-103.

17. He H, Ni J, Huang J. Molecular mechanisms of chemoresistance in osteosarcoma (Review). Oncology Letters. 2014;7(5):1352-1362.

18. Kim JS, Yu SK, Lee MH, et al. MicroRNA-205 directly regulates the tumor suppressor, interleukin-24, in human KB oral cancer cells. Molecules and Cells. 2013;35(1):17-24.

19. Zhang G, Hou X, Li Y, Zhao M. MiR-205 inhibits cell apoptosis by targeting phosphatase and tensin homolog deleted on chromosome ten in endometrial cancer Ishikawa cells. BMC Cancer. 2014;14:440.

20. Ratert N, Meyer HA, Jung M, et al. miRNA profiling identifies candidate mirnas for bladder cancer diagnosis and clinical outcome. The Journal of Molecular Diagnostics: JMD. 2013;15(5):695-705.

21. Qin AY, Zhang XW, Liu L, et al. MiR-205 in cancer: an angel or a devil? European Journal of Cell Biology. 2013;92(2):54-60.

22. Greene SB, Gunaratne PH, Hammond SM, Rosen JM. A putative role for microRNA-205 in mammary epithelial cell progenitors. Journal of Cell Science. 2010;123(Pt 4):606-618.

23. Dar AA, Majid S, de Semir D, Nosrati M, Bezrookove V, Kashani-SabetM. miRNA-205 suppresses melanoma cell proliferation and induces senescence via regulation of E2F1 protein. The Journal of Biological Chemistry. 2011;286(19):16606-16614.

24. Ason B, Darnell DK, Wittbrodt B, et al. Differences in vertebrate microRNA expression. Proceedings of the National Academy of Sciences of the United States of America. 2006;103(39):14385-14389.

25. Yi R, O'Carroll D, Pasolli HA, et al. Morphogenesis in skin is governed by discrete sets of differentially expressed microRNAs. Nature Genetics. 2006;38(3):356-362.

26. Gregory PA, Bert AG, Paterson EL, et al. The miR-200 family and miR-205 regulate epithelial to mesenchymal transition by targeting ZEB1 and SIP1. Nature Cell Biology. 2008;10(5):593-601.

27. Berber U, Yilmaz I, Narli G, Haholu A, Kucukodaci Z, Demirel D. miR-205 and miR-200c: Predictive Micro RNAs for Lymph Node Metastasis in Triple Negative Breast Cancer. Journal of Breast Cancer. 2014;17(2):143-148.

28. Mizobuchi H, Garcia-Castellano JM, Philip S, Healey JH, Gorlick R. Hypoxia markers in human osteosarcoma: an exploratory study. Clinical Orthopaedics and Related Research. 2008;466(9):2052-2059.

29. Rennel E, Waine E, Guan H, et al. The endogenous anti-angiogenic VEGF isoform, VEGF165b inhibits human tumour growth in mice. British Journal of Cancer. 2008;98(7):1250-1257.

30. Hicklin DJ, Ellis LM. Role of the vascular endothelial growth factor pathway in tumor growth and angiogenesis. Journal of clinical oncology: official journal of the American Society of Clinical Oncology. 2005;23(5):1011-1027.

31. Yang J, Yang D, Sun Y, et al. Genetic amplification of the vascular endothelial growth factor (VEGF) pathway genes, including VEGFA, in human osteosarcoma. Cancer. 2011;117(21):4925-4938.

\section{Dovepress}

patient perspectives such as quality of life, adherence and satisfaction The manuscript management system is completely online and includes a very quick and fair peer-review system, which is all easy to use. Visit http://www.dovepress.com/testimonials.php to read real quotes from published authors. 